\title{
Bifurcation and Solitary-Like Solutions for Compound KdV-Burgers-Type Equation
}

\author{
Yin Li, Ruiying Wei, and Guoming Jian \\ School of Mathematics and Statistics, Shaoguan University, Shaoguan 512005, China \\ Correspondence should be addressed to Ruiying Wei; weiruiying521@163.com and Guoming Jian; sdjgm@sgu.edu.cn
}

Received 22 October 2014; Accepted 22 January 2015

Academic Editor: Morteza Rafei

Copyright (C) 2015 Yin Li et al. This is an open access article distributed under the Creative Commons Attribution License, which permits unrestricted use, distribution, and reproduction in any medium, provided the original work is properly cited.

Firstly, based on the improved sub-ODE method and the bifurcation method of dynamical systems, we investigate the bifurcation of solitary waves in the compound KdV-Burgers-type equation. Secondly, numbers of solitary patterns solutions are given for each parameter condition and numerical simulations are used to display the dynamical characteristics. Finally, we obtain twelve solitary patterns solutions under some parameter conditions, such as the trigonometric function solutions and the hyperbolic function solutions.

\section{Introduction and Main Results}

Consider the following compound KdV-Burgers-type equation with nonlinear terms of any order:

$$
\begin{array}{r}
u_{t}+\left(a_{1}+a_{2} u^{p}+a_{3} u^{2 p}\right) u_{x}+a_{4} u_{x x x}=0, \\
p>0, \quad\left(a_{1}, a_{2}, a_{3}, a_{4}\right) \in R^{4},
\end{array}
$$

and the compound KdV-type equation with nonlinear terms of any order:

$$
\begin{array}{r}
u_{t}+a_{2} u^{p} u_{x}+a_{3} u^{2 p} u_{x}+a_{4} u_{x x x}=0, \\
p>0, \quad\left(a_{2}, a_{3}, a_{4}\right) \in R^{3} .
\end{array}
$$

These equations include a number of equations which have been studied by many authors [1-3]. Song et al. $[4,5]$ gave some solitary wave solutions and bifurcation phase portraits of (1) with $a_{4}=1, p$ being odd or even. It is necessary to point out that when we take the different parameters values, the following equations can be derived from (1).

If $a_{1}, a_{2}, a_{3}=$ constants and $a_{4}=1$, (1) becomes the equation

$$
\begin{array}{r}
u_{t}+a_{2} u^{p} u_{x}+a_{3} u^{2 p} u_{x}+u_{x x x}=0, \\
p>0, \quad\left(a_{2}, a_{3}, a_{4}\right) \in R^{3},
\end{array}
$$

which was studied in [1].
If $a_{1}=0, a_{2}=6, a_{3}= \pm 6$, and $p=a_{4}=1$, (1) becomes the $\mathrm{mKdV}$ and combined KdV equation [6]:

$$
u_{t}+6 u u_{x} \pm 6 u^{2} u_{x}+u_{x x x}=0 .
$$

If $p=a_{4}=1, a_{1}=0, a_{2}=2 \alpha$, and $a_{3}=-3 \beta$, (1) becomes Gardner's equation [7]:

$$
u_{t}+2 \alpha u u_{x}-3 \beta u^{2} u_{x}+u_{x x x}=0 .
$$

Biswas and Zerrad [7] also studied the soliton perturbation theory of (5).

If $a_{1} \neq 0, a_{2} \neq 0$ and $a_{3} \neq 0$, and $p=a_{4}=1$, (1) becomes the equation $[8]$

$$
u_{t}+\left(a_{1}+a_{2} u+a_{3} u^{2}\right) u_{x}+u_{x x x}=0 .
$$

Dey and Coffey $[9,10]$ considered the kink-profile solitary wave solutions for (1) with $p=1$ and $p=2$. The compound KdV-Burgers-type equation (2) with $p>1$ is a model for long-wave propagation in nonlinear media with dispersion and dissipation [11]; Coffey [3] considered soliton solutions, conservation laws, Backlund transformation, and other properties for (1) with $p=1$ and so on.

In this paper, we apply the bifurcation method of dynamical systems [12-23] and the improved sub-ODE method [2430] with the help of symbolic computation Mathematica and Maple to study solitary wave solutions of (1). 
TABLE 1: The property of singular point $O(0,0)$.

\begin{tabular}{lc}
\hline Conditions & Property of $O(0,0)$ \\
\hline$k>2 n+1$ & The origin is the saddle node. \\
$k<2 n+1$, or $\phi=0$ & The origin is a cusp. \\
\hline
\end{tabular}

TABLE 2: The property of singular point $O(0,0)$.

\begin{tabular}{lc}
\hline Conditions & Property of $O(0,0)$ \\
\hline$n$ even, $k>2 \lambda+1$ or $n$ even, & Stable node \\
$k=2 \lambda+1, b_{n}^{2}+4 k(\lambda+1) \geq 0$ & \\
$n$ odd, $k=2 \lambda+1, b_{n}^{2}+4 k(\lambda+1) \geq 0$ or $n$ & Elliptic saddle \\
odd, $k>2 \lambda+1$ & Focus or center \\
$k=2 \lambda+1, b_{n}^{2}+4 k(\lambda+1)<0$ or $k<2 \lambda+1$ & \\
\hline
\end{tabular}

Based on the bifurcation method and the improved sub-ODE method with the help of symbolic computation Mathematica, the bifurcation for (1) is derived by use of a proper transformation. To our knowledge, this type of transformation obtained has not been ever seen before in the literature. Then based on this transformation, some new solutions for (1) and (2) are found.

This paper is organized as follows. In Section 2, we derive the bifurcations of phase portraits of (1). In Section 3, we give the main results via the sub-ODE method. In Section 4 , the main results and some exact travelling wave solutions for (1) are obtained. Conclusions are given in the last section.

\section{Bifurcations of Phase Portraits of (1)}

In this section, we discuss some bifurcation phase portraits of (1). To study dynamical behavior of (1), we first need to give the lemmas $[21,31]$ in this section.

Lemma 1. Let $(0,0)$ be a nilpotent singular point of the vector field $(y+P(x, y), Q(x, y))$, where $P$ and $Q$ are analytic functions in a neighborhood of the origin at least with quadratic terms in the variables $x$ and $y$. Let $y=f(x)$ be the solution of the equation $y+P(x, y)=0$ in a neighborhood of $(0,0)$. Assume that the development of the function $Q(x, f(x))$ is of the form $a_{k} x^{k}+$ Hot (higher order terms) and $\phi(x)=(d P / d x+$ $d Q / d y)(x, f(x))=b_{n} x^{n}+$ Hot with $a_{k} \neq 0, k \geq 2$, and $n \geq 1$. For $k=2 n$ or $k=2 n+1$, one gives the property of singular point $o(0,0)$ as in Tables 1 and 2 .

Case 1. For this case, $k$ is even.

Case 2. For this case, $k$ is odd and $a_{k}<0$.

To this end, substituting $u=\phi(\xi)$ with $\xi=x-V t$ into (1), we have

$$
-V \phi^{\prime}+a_{1} \phi^{\prime}+a_{2} \phi^{p} \phi^{\prime}+a_{3} \phi^{2 p} \phi^{\prime}+a_{4} \phi^{\prime \prime \prime}=0 .
$$

If $|\xi|$ tends to $\infty$, we obtain the solitary wave solutions and kink wave solutions tend to some constants. This implies that $\phi^{\prime}, \phi^{\prime \prime}$, and $\phi^{\prime \prime \prime}$ tend to zero, when $|\xi|$ tends to $\infty$. Integrating (7) once we get

$$
\left(-V+a_{1}\right) \phi+\frac{a_{2}}{p+1} \phi^{p+1}+\frac{a_{3}}{2 p+1} \phi^{2 p+1}+a_{4} \phi^{\prime \prime}=g
$$

where $g$ is the constant of integration. Letting $y=\phi^{\prime}$, we obtain a planar system:

$$
\frac{d \phi}{d \xi}=y
$$

$$
\frac{d y}{d \xi}=f(\phi)-g
$$

where $f(\phi)=-\left(a_{3} /(2 p+1) a_{4}\right) \phi^{2 p+1}-\left(a_{2} /(p+1) a_{4}\right) \phi^{p+1}+$ $\left(\left(V-a_{1}\right) / a_{4}\right) \phi$.

System (9) has the following first integral:

$$
\begin{aligned}
H(\phi, y)= & \frac{1}{2} y^{2}+\frac{a_{3}}{(2 p+1)(2 p+2) a_{4}} \phi^{2 p+2} \\
& +\frac{a_{2}}{(p+1)(p+2) a_{4}} \phi^{p+2} \\
& +\frac{-V+a_{1}}{2 a_{4}} \phi^{2}-\frac{g \phi}{a_{4}}=h,
\end{aligned}
$$

where $h$ is the constant of integration. To study the distribution of singular points of system (9), we need to investigate the zero points of $f(\phi)$. If $g=0, p$ is odd, $a_{3}, a_{4} \neq 0$, and $\Delta>0$, then we get three zero points of $f(\phi)$ as

$$
\begin{aligned}
& \phi_{1}=0 \\
& \phi_{2}=\left[-\frac{(2 p+1) a_{2}-a_{4} \sqrt{\Delta}}{2 a_{3}(p+1)}\right]^{1 / p}, \\
& \phi_{3}=\left[-\frac{(2 p+1) a_{2}+a_{4} \sqrt{\Delta}}{2 a_{3}(p+1)}\right]^{1 / p},
\end{aligned}
$$

where $\Delta=\left(a_{2}^{2} / a_{4}^{2}(p+1)\right)^{2}+\left(4\left(V-a_{1}\right) a_{3} / a_{4}^{2}(2 p+1)\right)$. Suppose $(\phi, 0)$ is a singular point of system $(9)$. Let

$$
M(\phi, 0)=\left(\begin{array}{cc}
0 & 1 \\
-\frac{a_{3} \phi^{2 p}+a_{2} \phi^{p}+\left(-V+a_{1}\right)}{a_{4}} & 0
\end{array}\right)
$$

be the coefficient matrix of the linearized system of (9). Then the eigenvalue of $M(\phi, 0)$ at $(\phi, 0)$ is

$$
\lambda_{ \pm}(\phi, 0)= \pm \sqrt{-\frac{a_{3} \phi^{2 p}+a_{2} \phi^{p}+\left(-V+a_{1}\right)}{a_{4}}}= \pm \sqrt{f^{\prime}(\phi)} .
$$


TABLE 3: The local bifurcation phase portraits of system (9).

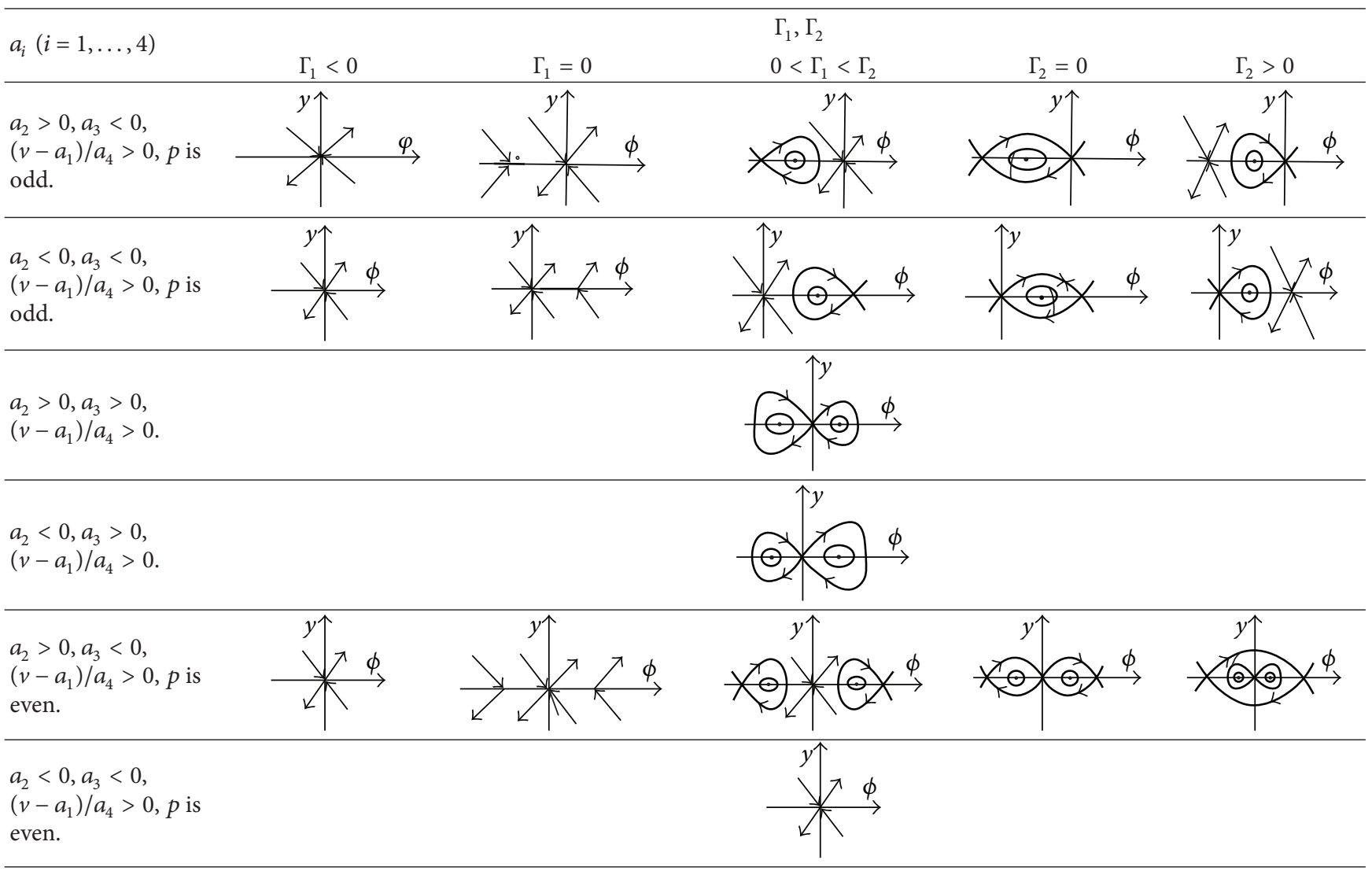

According to Lemma 1 and the theory of dynamical systems $[4,5,12-16]$, we obtain the following conclusions.

(i) If $f^{\prime}\left(\phi_{i}\right)>0,\left(\phi_{i}, 0\right)$ is a saddle point.

(ii) If $f^{\prime}\left(\phi_{i}\right)=0,\left(\phi_{i}, 0\right)$ is a degenerate saddle point.

(iii) If $f^{\prime}\left(\phi_{i}\right)<0,\left(\phi_{i}, 0\right)$ is a center point. Solving equations $f\left(\phi_{i}\right)=0$ and $H\left(\phi_{i}, 0\right)=0$, respectively, we get two bifurcation curves $\Gamma_{1}$ and $\Gamma_{2}$ as follows:

$$
\begin{aligned}
& \Gamma_{1}: a_{3}=-\frac{2 p+1}{4\left(V-a_{1}\right)(p+1)^{2}} a_{2}^{2}, \\
& \Gamma_{2}: a_{3}=-\frac{2 p+1}{\left(V-a_{1}\right)(p+1)(P+2)^{2}} a_{2}^{2} .
\end{aligned}
$$

From the above analysis, we obtain the bifurcation phase portraits of system (9) as in Table 3.

\section{The Improved Method and Statement of the Main Results}

In this section, we will give some exact parametric representations of travelling wave solutions of (1). To study solitary wave solutions and the qualitative behavior of system (1), we first need to introduce the improved method based on [25] as follows. Suppose a nonlinear PDE,

$$
p\left(u, u_{t}, u_{x}, u_{x t}, u_{t t}, u_{x x}, \ldots\right)=0,
$$

where $P$ is a polynomial in its arguments.
Step 1 (reduce NPDE to nonlinear ODE). By taking the transformation $u(x, t)=u(\xi), \xi=x-V t$, where $V$ is arbitrary nonzero constants, and (15) transform it to the ordinary differential equation reduces to be

$$
G\left(u^{\prime}, u^{\prime \prime}, u^{\prime \prime \prime}, \ldots\right)=0 .
$$

Step 2 (determine the parameters). Determine the highest order nonlinear term and the linear term of the highest order term in (15) or (16). Then, in the resulting terms, balance the highest order nonlinear term and the linear term of the highest order term; we get a balance constant $k$ ( $k$ is usually a positive integer). If $k$ is a negative integer or a fraction, we make the following transformation:

$$
u(\xi)=v^{k}(\xi) .
$$

We obtain the solutions of (15) to be as the following forms.

Case 1. If $R \neq 0$ in (19)-(20),

$$
u(\xi)=A_{0}+\sum_{i=1}^{m} \sigma^{i-1}\left[A_{i} \sigma(\xi)+B_{i} \tau(\xi)\right],
$$

where $\sigma(\xi)$ and $\tau(\xi)$ satisfy the following equations:

$$
\begin{array}{r}
\sigma^{\prime}(\xi)=\epsilon \sigma(\xi) \tau(\xi), \quad \tau^{\prime}(\xi)=R+\epsilon \tau^{2}(\xi)-\mu \sigma(\xi), \\
\epsilon= \pm 1, \quad R, \mu=\text { constant }
\end{array}
$$


which admits the first integral with $R \neq 0$,

$$
\tau^{2}(\xi)=-\epsilon\left[R-2 \mu \sigma(\xi)+\frac{\mu^{2}-1}{R} \sigma^{2}(\xi)\right], \quad(R \neq 0),
$$

where $I=d / \xi$. When $\epsilon=-1, R=1$, and $\mu \rightarrow \mu / K,(20)$ becomes a projective Riccati equation [25].

Case 2. If $R=\mu=0$ in (19)-(20),

$$
u(\xi)=\sum_{i=0}^{k} A_{i} \tau^{i}(\xi)
$$

where $\tau^{\prime}(\xi)=\tau^{2}(\xi)$.

Step 3. (I) If $R \neq 0$, substitute (18) along with conditions (19)(20) into (15).

(II) If $R=\mu=0$, substituting (21) along with $\tau^{\prime}(\xi)=\tau^{2}(\xi)$ into (15) yields a set of algebraic equations for $\sigma^{j}(\xi) \tau^{i}(\xi)$, $(j=0,1, \ldots ; i=0,1)\left(\tau^{l}(\xi), l=0,1, \ldots\right)$. Setting the coefficients of these terms $\sigma^{j} \tau^{i}$ (or $\tau^{l}(\xi)$ ) to zero yields a set of overdetermined algebraic equations in $V, A_{i}, B_{i}, R$, and $\mu$.

Step 4. According to the theory of dynamical systems with the aid of Mathematica, solving the above set of equations yields the values of $A_{i}, B_{i}, R$, and $\lambda$.

Step 5. We know that (15) admits the following solutions.

Case 1. If $\epsilon=-1, R \neq 0$,

$$
\begin{array}{ll}
\sigma_{1}(\xi)=\frac{R \operatorname{sech}(\sqrt{R} \xi)}{\mu \operatorname{sech}(\sqrt{R} \xi)+1}, & \tau_{1}(\xi)=\frac{\sqrt{R} \tanh (\sqrt{R} \xi)}{\mu \operatorname{sech}(\sqrt{R} \xi)+1}, \\
\sigma_{2}(\xi)=\frac{R \operatorname{csch}(\sqrt{R} \xi)}{\mu \operatorname{csch}(\sqrt{R} \xi)+1}, & \tau_{2}(\xi)=\frac{\sqrt{R} \operatorname{coth}(\sqrt{R} \xi)}{\mu \operatorname{csch}(\sqrt{R} \xi)+1} .
\end{array}
$$

Case 2. If $\epsilon=1, R \neq 0$,

$$
\begin{gathered}
\sigma_{3}(\xi)=\frac{R \sec (\sqrt{R} \xi)}{\mu \sec (\sqrt{R} \xi)+1}, \quad \tau_{3}(\xi)=\frac{\sqrt{R} \tan (\sqrt{R} \xi)}{\mu \sec (\sqrt{R} \xi)+1}, \\
\sigma_{4}(\xi)=\frac{R \csc (\sqrt{R} \xi)}{\mu \csc (\sqrt{R} \xi)+1}, \\
\tau_{4}(\xi)=-\frac{\sqrt{R} \cot (\sqrt{R} \xi)}{\mu \csc (\sqrt{R} \xi)+1} .
\end{gathered}
$$

Case 3. If $R=\mu=0$,

$$
\sigma_{5}(\xi)=\frac{c}{\xi}=c \epsilon \tau_{5}(\xi), \quad \tau_{5}(\xi)=\frac{1}{\epsilon \xi},
$$

where $c$ is a constant. According to (18), (21), (22)-(24), and the conclusions in Step 4, we can obtain many solutions for (15).

Under the above method and some parameter conditions, exact solitary wave solutions are obtained. Our results are as follows.
Proposition 2. (1) If $V=a_{1}-\left(a_{4} / a_{3}\right)\left((2 p+1) a_{2}^{2} /(2+p)^{2}\right)$, (1) has two solitary wave trigonometric function solutions:

$$
\begin{aligned}
& u_{1}(x, t)=\left( \pm \sqrt{R} B_{1}+B_{1} \sqrt{R} \tanh (\sqrt{R}(x-V t))\right)^{1 / p}, \\
& u_{2}(x, t)=\left( \pm \sqrt{R} B_{1}+B_{1} \sqrt{R} \operatorname{coth}(\sqrt{R}(x-V t))\right)^{1 / p},
\end{aligned}
$$

where $R=-(2 p+1) p^{2} a_{2}^{2} / 4 a_{3}(2+p)^{2}$ and $B_{1}=$ $\pm \sqrt{a_{4}(p+1)(2 p+1) /-p^{2} a_{3}}$.

(2) If $V=\left(R^{2} A_{1}^{2} a_{3}+2 a_{1} p^{2}+3 a_{1} p+a_{1}\right) /(p+1)(2 p+1)$, (1) has two solitary wave solutions:

$$
\begin{aligned}
& u_{3}(x, t)=\left(A_{1} R \operatorname{sech}(\sqrt{R(x-V t)})\right)^{1 / p}, \\
& u_{4}(x, t)=\left(A_{1} R \operatorname{csch}(\sqrt{R(x-V t)})\right)^{1 / p},
\end{aligned}
$$

where $R=a_{4}(2 p+1)(p+1) / a_{3} A_{1}^{2} p^{2}$ and $A_{1}$ is arbitrary constant.

Proposition 3. (1) If $V=-\left(-2 a_{1} p^{2}-3 a_{1} p-a_{1}+a_{1}(p+\right.$ 1) $\left.(2 p+1) R / p^{2} a_{3}\right)(p+1)^{-1}(2 p+1)^{-1}$, (1) has two solitary wave solutions:

$$
\begin{aligned}
& u_{5}(x, t)=\left(\mp \sqrt{R} B_{1}+B_{1} \frac{\sqrt{R} \tanh (\sqrt{R}(x-V t))}{\operatorname{sech}(\sqrt{R}(x-V t))+1}\right)^{1 / p}, \\
& u_{6}(x, t)=\left(\mp \sqrt{R} B_{1}+B_{1} \frac{\sqrt{R} \operatorname{coth}(\sqrt{R}(x-V t))}{\operatorname{csch}(\sqrt{R}(x-V t))+1}\right)^{1 / p},
\end{aligned}
$$

where $R=(1 / 4)\left(a_{2}{ }^{2}(2 p+1)^{2} / a_{3}{ }^{2} B_{1}{ }^{2}(2+p)^{2}\right), \quad \mu=1$, and $B_{1}= \pm(1 / 2)\left(\sqrt{-a_{3} a_{4}\left(2 p^{2}+3 p+1\right)} / a_{3} p\right)$.

(2) If $V=\left(R a_{2} A_{1}+3 a_{1} p+a_{1} p^{2}+2 a_{1}\right) /(2+p)(p+1)$, (1) has two solitary wave solutions:

$$
\begin{aligned}
& u_{7}(x, t)=\left(A_{1} R \frac{\sec (\sqrt{R}(x-V t))}{\sec (\sqrt{R}(x-V t))+1}\right)^{1 / p}, \\
& u_{8}(x, t)=\left(A_{1} R \frac{\operatorname{sech}(\sqrt{R}(x-V t))}{\operatorname{csch}(\sqrt{R}(x-V t))+1}\right)^{1 / p},
\end{aligned}
$$

where $A_{1}=-a_{4}(p+1)(2+p) / p^{2} a_{2}, \mu=1$, and $R$ is arbitrary constant. 
Proposition 4. (1) If $V=a_{1}, a_{4}(p+1)(2 p+1)=0$, (1) has two solitary wave hyperbolic function solutions:

$$
\begin{aligned}
& u_{9}(x, t)=\left(\mp \sqrt{R} B_{1} \pm \frac{B_{1}}{\sqrt{R}} \frac{R \operatorname{sech}(\sqrt{R}(x-V t))}{\operatorname{sech}(\sqrt{R}(x-V t))+1}\right. \\
& \left.+B_{1} \frac{\sqrt{R} \tanh (\sqrt{R}(x-V t))}{\operatorname{sech}(\sqrt{R}(x-V t))}\right)^{1 / p}, \\
& u_{10}(x, t)=\left(\mp \sqrt{R} B_{1} \pm \frac{B_{1}}{\sqrt{R}} \frac{R \operatorname{csch}(\sqrt{R}(x-V t))}{\operatorname{csch}(\sqrt{R}(x-V t))+1}\right. \\
& \left.+B_{1} \frac{\sqrt{R} \operatorname{coth}(\sqrt{R}(x-V t))}{\operatorname{csch}(\sqrt{R}(x-V t))}\right)^{1 / p},
\end{aligned}
$$

where $\mu=-1$ and $B_{1}$ and $R$ are arbitrary constants.

(2) If $V=-\left(R^{2} A_{1}{ }^{2} a_{3}-2 a_{1} p^{2} \mu^{2}+2 a_{1} p^{2}-3 a_{1} p \mu^{2}+3 a_{1} p-\right.$ $\left.a_{1} \mu^{2}+a_{1}\right) /\left(\mu^{2}-1\right)(p+1)(2 p+1)$, (1) has two solitary wave solutions:

$$
\begin{aligned}
& u_{11}(x, t)=\left(A_{1} R \frac{\sec (\sqrt{R}(x-V t))}{\mu \sec (\sqrt{R}(x-V t))+1}\right)^{1 / p}, \\
& u_{12}(x, t)=\left(A_{1} R \frac{\csc (\sqrt{R}(x-V t))}{\mu \csc (\sqrt{R}(x-V t))+1}\right)^{1 / p},
\end{aligned}
$$

where $R=-a_{4}(p+1)(2 p+1)\left(\mu^{2}-1\right) / p^{2} A_{1}^{2} a_{3}, A_{1}=$ $-a_{2}\left(2 p \mu^{2}-2 p+\mu^{2}-1\right) /(2+p) R \mu a_{3}$ and $\mu$ is arbitrary constant.

Proposition 5. If $\left(V-a_{1}\right) / a_{4}>0, a_{3}>0$, (1) has two solitary wave solutions:

$$
\begin{aligned}
& u_{13}(x, t)=\left(-\frac{(p+1)(p+2)\left(V-a_{1}\right)}{a_{4}\left(A_{1} \cosh (R(x-V t))\right)-a_{2}}\right)^{1 / p}, \\
& u_{14}(x, t)=\left(\frac{(p+1)(p+2)\left(V-a_{1}\right)}{a_{4}\left(A_{1} \cosh (R(x-V t))\right)+a_{2}}\right)^{1 / p},
\end{aligned}
$$

where

$$
\begin{gathered}
A_{1}=-\sqrt{\frac{(2 p+1) a_{2}^{2}+(p+1)(p+2)^{2}\left(V-a_{1}\right) a_{3}}{(2 p+1) a_{4}}}, \\
R=p \sqrt{\frac{c-a_{1}}{a_{4}}} .
\end{gathered}
$$

\section{The Derivations of Theorem}

4.1. The Derivations of Proposition Using the Improved Sub$O D E$ Method. In this section, we make the travelling transformation to (1) $u(x, t)=\phi(\xi), \xi=x-V t$, where $V$ is a constant to be determined later, and thus (1) becomes (8). Integrating (8) once with regard to $\xi$, we obtain

$$
\left(-V+a_{1}\right) \phi+\frac{a_{2}}{p+1} \phi^{p+1}+\frac{a_{3}}{2 p+1} \phi^{2 p+1}+a_{4} \phi^{\prime \prime}=0
$$

with the integration constants taken to be zero.
According to Step 1 in Section 3, if $a_{1} \neq 0, a_{2} \neq 0, a_{3} \neq 0$, $a_{4} \neq 0$, and $p \neq 0$, by balancing $\phi^{2 p+1}$ and $\phi^{\prime \prime}$ in (34), we get $k=1 / p$. Therefore, we make the following transformation:

$$
\phi(\xi)=\varphi^{1 / p}(\xi)
$$

then substituting (34) into (35) yields

$$
\begin{gathered}
p^{2} b_{0} \varphi^{2}+a_{2} p^{2}(2 p+1) \varphi^{3}+a_{3}(p+1) p^{2} \varphi^{4} \\
+b_{1}(1-p)\left(\varphi^{\prime}\right)^{2}+b_{1} p \varphi^{\prime \prime} \varphi=0,
\end{gathered}
$$

where

$$
\begin{aligned}
& b_{0}=(p+1)(2 p+1)\left(-V+a_{1}\right), \\
& b_{1}=a_{4}(p+1)(2 p+1) .
\end{aligned}
$$

According to Step 1 in Section 3, by balancing $\varphi(\xi) \varphi^{\prime \prime}(\xi)$ (or $\varphi^{\prime 2}(\xi)$ ) and $\varphi^{4}(\xi)$ in (36), we get $k=1$. Therefore, we suppose that (37) has the following formal solutions:

$$
\varphi(\xi)=A_{0}+A_{1} \sigma(\xi)+B_{1} \tau(\xi)
$$

where $\sigma(\xi), \tau(\xi)$ satisfy (19)-(20), where $A_{0}, A_{1}, B_{1}$ are constants to be determined later.

With the aid of Mathematica and Maple, substituting (38) along with (18)-(19) into (36) yields a set of algebraic equations for $\sigma^{j}(\xi) \tau^{i}(\xi)(j=0,1, \ldots ; i=0,1)$. Setting the coefficients of these terms $\sigma^{j}(\xi) \tau^{i}(\xi)$ to zero yields a set of overdetermined algebraic equations with respect to $A_{0}, A_{1}$, $B_{1}, R$, and $V$.

Case 1. Consider

$$
\begin{gathered}
\mu=A_{1}=0, \quad b_{0}=4 B_{1}^{2} R a_{3}, \\
b_{1}=-p^{2} B_{1}^{2} a_{3}, \quad A_{0}= \pm \sqrt{R} B_{1}, \\
a_{2}= \pm 2 \frac{(2+p) a_{3} \sqrt{R} B_{1}}{2 p+1} .
\end{gathered}
$$

Case 2. Consider

$$
\begin{gathered}
\mu=0, \quad b_{1}=-4 p^{2} B_{1}{ }^{2} a_{3}, \quad b_{0}=4 B_{1}{ }^{2} R a_{3}, \\
A_{1}= \pm \frac{B_{1}}{\sqrt{-R}}, \quad a_{2}=-2 \frac{(2+p) a_{3} \sqrt{R} B_{1}}{2 p+1}, \\
A_{0}=\sqrt{R} B_{1} .
\end{gathered}
$$

Case 3. Consider

$$
\begin{gathered}
\mu=0, \quad A_{1}= \pm \frac{B_{1}}{\sqrt{-R}}, \quad b_{0}=4 B_{1}{ }^{2} R a_{3}, \\
b_{1}=-4 p^{2} B_{1}{ }^{2} a_{3} \quad a_{2}=2 \frac{(2+p) a_{3} \sqrt{R} B_{1}}{2 p+1}, \\
A_{0}=-\sqrt{R} B_{1} .
\end{gathered}
$$


Case 4. Consider

$$
\begin{gathered}
\mu=A_{0}=a_{2}=B_{1}=0, \quad b_{1}=a_{3} A_{1}{ }^{2} p^{2} R, \\
b_{0}=-R^{2} A_{1}{ }^{2} a_{3},
\end{gathered}
$$

where $A_{1}$ is an arbitrary constant.

Case 5. Consider

$$
\begin{gathered}
\mu= \pm 1, \quad A_{1}=0, \quad b_{1}= \pm 4 p^{2} B_{1}{ }^{2} a_{3}, \\
b_{0}=4 B_{1}{ }^{2} R a_{3}, \quad a_{2}= \pm 2 \frac{(2+p) a_{3} \sqrt{R} B_{1}}{2 p+1}, \\
A_{0}=\mp \sqrt{R} B_{1} .
\end{gathered}
$$

Case 6. Consider

$$
\begin{gathered}
\mu= \pm 1, \quad b_{0}=b_{1}=a_{2}=a_{3}=0, \\
A_{1}= \pm \frac{B_{1}}{\sqrt{R}}, \quad A_{0}= \pm \sqrt{R} B_{1} .
\end{gathered}
$$

Case 7. Consider

$$
\begin{gathered}
\mu= \pm 1, \quad A_{0}=B_{1}=a_{3}=0 \\
b_{1}= \pm \frac{A_{1} a_{2} p^{2}(2 p+1)}{2+p}, \quad b_{0}=\mp \frac{A_{1} R a_{2}(2 p+1)}{2+p} .
\end{gathered}
$$

Case 8. Consider

$$
\begin{gathered}
A_{0}=B_{1}=0, \quad a_{2}=-\frac{(2+p) R \mu a_{3} A_{1}}{2 p \mu^{2}-2 p+\mu^{2}-1}, \\
b_{0}=\frac{R^{2} A_{1}{ }^{2} a_{3}}{\mu^{2}-1}, \quad b_{1}=-\frac{a_{3} A_{1}{ }^{2} p^{2} R}{\mu^{2}-1},
\end{gathered}
$$

where $\mu \neq 1$ and $A_{1}$ are arbitrary constants and $b_{0}$ and $b_{1}$ are the same as (37).

Solving the overdetermined algebraic equations, we get Propositions 2-5.

4.2. The Derivations of Proposition Using the Integral Method. In the following, we study the derivations of theorem using the integral method $[4,5,13-16]$.

If $g=0,(9)$ reduces to the following system:

$$
\begin{gathered}
\frac{d \varphi}{d \xi}=y \\
\frac{d y}{d \xi}=-\left((p+1)(2 p+1)\left(-V+a_{1}\right) \varphi\right. \\
\left.+a_{3}(p+1) \varphi^{2 p+1}+a_{2}(2 p+1) \varphi^{p+1}\right) \\
\cdot\left(a_{4}(p+1)(2 p+1)\right)^{-1} .
\end{gathered}
$$

From Table 3, we obtain two homoclinic orbits $\Gamma_{i}(i=1,2)$ of (9).
In $\varphi-y$ plane, $L_{1}$ is described as

$$
\begin{array}{r}
y^{2}=\varphi^{2}\left(\frac{V-a_{1}}{a_{4}}-\frac{2 a_{2}}{(p+1)(p+2) a_{4}} \varphi^{p}\right. \\
\left.-\frac{a_{3}}{(2 p+1)(p+1) a_{4}} \varphi^{2 p}\right), \\
\varphi \in\left[\varphi_{1}, \varphi_{2}\right],
\end{array}
$$

where

$$
\begin{aligned}
& \varphi_{1}=\left[-\frac{(2 p+1) a_{2}-a_{4} \sqrt{\Delta}}{2 a_{3}(p+1)}\right]^{1 / p}, \\
& \varphi_{2}=\left[-\frac{(2 p+1) a_{2}+a_{4} \sqrt{\Delta}}{2 a_{3}(p+1)}\right]^{1 / p}, \\
& \Delta=\frac{a_{2}^{2}}{a_{4}^{2}(p+1)^{2}}+\frac{4\left(V-a_{1}\right) a_{3}}{a_{4}^{2}(2 p+1)} .
\end{aligned}
$$

We obtain the results by substituting (48) into $d \varphi / d \xi=y$ :

$$
\begin{aligned}
\pm\left(1 \cdot \left(\varphi ^ { 2 } \left(\frac{V-a_{1}}{a_{4}}-\frac{2 a_{2}}{(p+1)(p+2) a_{4}} \varphi^{p}\right.\right.\right. \\
\\
\left.\left.\left.\quad-\frac{a_{3}}{(2 p+1)(p+1) a_{4}} \varphi^{2 p}\right)\right)^{-1}\right)^{1 / 2} d \varphi=d \xi .
\end{aligned}
$$

If $\varphi(0)=\varphi_{1}$, then, integrating (50) along homoclinic orbit $L_{1}$, we have

$$
\begin{aligned}
& \int_{\varphi_{1}}^{\varphi}\left(d w \cdot \left(w ^ { 2 } \left(\frac{V-a_{1}}{a_{4}}-\frac{2 a_{2}}{(p+1)(p+2) a_{4}} w^{p}\right.\right.\right. \\
& \left.\left.\left.-\frac{a_{3}}{(2 p+1)(p+1) a_{4}} w^{2 p}\right)\right)^{-1}\right) \\
& =\int_{0}^{\xi} d w \quad \xi<0, \\
& -\int_{\varphi}^{\varphi_{1}}\left(d w \cdot \left(w ^ { 2 } \left(\frac{V-a_{1}}{a_{4}}-\frac{2 a_{2}}{(p+1)(p+2) a_{4}} w^{p}\right.\right.\right. \\
& =\int_{0}^{\xi} d w \quad \xi>0 .
\end{aligned}
$$

We obtain by completing the above two integrals

$$
\varphi_{4}(x, t)=\left(-\frac{(p+1)(p+2)\left(V-a_{1}\right)}{a_{4}\left(A_{1} \cosh (R(x-V t))\right)-a_{2}}\right)^{1 / p},
$$


where

$$
\begin{gathered}
A_{1}=-\sqrt{\frac{(2 p+1) a_{2}^{2}+(p+1)(p+2)^{2}\left(V-a_{1}\right) a_{3}}{(2 p+1) a_{4}}}, \\
R=p \sqrt{\frac{c-a_{1}}{a_{4}}} .
\end{gathered}
$$

We obtain $u_{13}(x, t)$ as formula (31) via (53).

If $\varphi(0)=\varphi_{2}$, integrating (50) along homoclinic orbit $L_{2}$, we have

$$
\begin{gathered}
\int_{\varphi_{2}}^{\varphi}\left(d w \cdot \left(w ^ { 2 } \left(\frac{V-a_{1}}{a_{4}}-\frac{2 a_{2}}{(p+1)(p+2) a_{4}} w^{p}\right.\right.\right. \\
\left.\left.\left.-\frac{a_{3}}{(2 p+1)(p+1) a_{4}} w^{2 p}\right)\right)^{-1}\right) \\
=\int_{0}^{\xi} d w \quad \xi>0, \\
-\int_{\varphi}^{\varphi_{2}}\left(d w \cdot \left(w ^ { 2 } \left(\frac{V-a_{1}}{a_{4}}-\frac{2 a_{2}}{(p+1)(p+2) a_{4}} w^{p}\right.\right.\right. \\
=\int_{0}^{\xi} d w \quad \xi<0 .
\end{gathered}
$$

We obtain by completing the above two integrals

$$
\varphi_{5}(x, t)=\left(\frac{(p+1)(p+2)\left(V-a_{1}\right)}{a_{4}\left(A_{1} \cosh (R(x-V t))\right)+a_{2}}\right)^{1 / p}
$$

where

$$
\begin{gathered}
A_{1}=-\sqrt{\frac{(2 p+1) a_{2}^{2}+(p+1)(p+2)^{2}\left(V-a_{1}\right) a_{3}}{(2 p+1) a_{4}},} \\
R=p \sqrt{\frac{c-a_{1}}{a_{4}}} .
\end{gathered}
$$

Our results (52) and (55) can be compared with the result of Song et al. [5] by assigning special values to the arbitrary parameters involved.

From (52), (55), and traveling wave transformation (34), we get solitary wave solutions $u_{13}(x, y, t), u_{14}(x, y, t)$ just as expressed in (31) and (32).

Similarly, we can get other solutions. Here we complete the derivation of Propositions 2-4.

\section{Conclusion}

In this paper, we were devoted to investigate the dynamical survey and the phase orbits of the compound KdV-Burgerstype equation via the improved sub-ODE method and the integral method. Some solitary wave solutions are given for each parameter condition. At the same time, the method can be widely applied to other nonlinear equations. Not only do we obtain the explicit solitary wave solutions but also we can get other kinds of solutions such as the trigonometric function solutions, the hyperbolic function solutions, and the rational solutions.

\section{Conflict of Interests}

The authors declare that there is no conflict of interests regarding the publication of this paper.

\section{Acknowledgments}

The authors are grateful to Professor Yulin Zhao and Professor Zheng-an Yao of Sun Yat-sen University and Professors Y. Chen of East China Normal University, B. Li of NingBo University, and C. L. Zheng and Shaoyong Li of Shaoguan University for their fruitful help and discussions. The authors are also in debt to express thanks to the referees for valuable suggestions and for their positive comments. This work is supported by the NSF of China (no. 11171355), Guangdong Provincial Culture of Seedling of China (no. 2013LYM0081), Guangdong Provincial NSF of China (no. S2012010010069), Guangdong Provincial Department of Education research platform project (Guangdong Young Creative Talents (no. 2014208)). The Shaoguan Science and Technology Foundation (no. 313140546), and Science Foundation of Shaoguan University.

\section{References}

[1] M. Wadati, "Wave propagation in nonlinear lattice," Journal of the Physical Society of Japan, vol. 38, pp. 673-679, 1975.

[2] R. L. Pego, "The linear stability of traveling waves to the compound KdV-Burgers-type equation," Journal of Physics D, vol. 67, pp. 45-56, 1993.

[3] M. W. Coffey, "New exact traveling wave solutions for compound KdV-Burgers-type equation," SIAM Journal on Applied Mathematics, vol. 50, pp. 1580-1589, 1990.

[4] M. Song and S. Shao, "Exact solitary wave solutions of the generalized $(2+1)$ dimensional Boussinesq equation," Applied Mathematics and Computation, vol. 217, no. 7, pp. 3557-3563, 2010.

[5] M. Song, X. Hou, and J. Cao, "Solitary wave solutions and kink wave solutions for a generalized KDV-mKDV equation," Applied Mathematics and Computation, vol. 217, no. 12, pp. 5942-5948, 2011.

[6] H. Wu, Y. Zeng, and T. Fan, "On the extended KdV equation with self-consistent sources," Physics Letters A, vol. 370, no. 5-6, pp. 477-484, 2007.

[7] A. Biswas and E. Zerrad, "Soliton perturbation theory for the Gardner equation," Advanced Studies in Theoretical Physics, vol. 2, no. 16, pp. 787-794, 2008.

[8] J. Zhang, F. Wu, and J. Shi, "Simple soliton solution method for the combined KdV and MKdV equation," International Journal of Theoretical Physics, vol. 39, no. 6, pp. 1697-1702, 2000. 
[9] B. Dey, "Domain wall solutions of KdV-like equations with higher order nonlinearity," Journal of Physics. A. Mathematical and General, vol. 19, no. 1, pp. L9-L15, 1986.

[10] M. W. Coffey, "On series expansions giving closed-form solutions of Korteweg-de Vries-like equations," SIAM Journal on Applied Mathematics, vol. 50, no. 6, pp. 1580-1592, 1990.

[11] B. Li, Y. Chen, and H. Q. Zhang, "Auto-Bäcklund transformation and exact solutions for compound KDV-type and compound KDV-burgers-type equations with nonlinear terms of any order," Physics Letters A, vol. 305, no. 6, pp. 377-382, 2002.

[12] M. A. Abdou, "Exact periodic wave solutions to some nonlinear evolution equations," International Journal of Nonlinear Science, vol. 6, no. 2, pp. 145-153, 2008.

[13] J. B. Li and Z. R. Liu, "Smooth and non-smooth traveling waves in a nonlinearly dispersive equation," Applied Mathematical Modelling, vol. 25, no. 1, pp. 41-56, 2000.

[14] Z. Liu and T. Qian, "Peakons and their bifurcation in a generalized Camassa-Holm equation," International Journal of Bifurcation and Chaos in Applied Sciences and Engineering, vol. 11, no. 3, pp. 781-792, 2001.

[15] Z. R. Liu and C. X. Yang, "The application of bifurcation method to a higher-order KdV equation," Journal of Mathematical Analysis and Applications, vol. 275, no. 1, pp. 1-12, 2002.

[16] W.-L. Zhang, "Solitary wave solutions and kink wave solutions for a generalized PC equation," Acta Mathematicae Applicatae Sinica: English Series, vol. 21, no. 1, pp. 125-134, 2005.

[17] M. Y. Tang and W. L. Zhang, "Four types of bounded wave solutions of $\mathrm{CH}-\gamma$ equation," Science in China. Series A: Mathematics, vol. 50, no. 1, pp. 132-152, 2007.

[18] Z. R. Liu and Z. Y. Ouyang, "A note on solitary waves for modified forms of Camassa-Holm and Degasperis-Procesi equations," Physics Letters A, vol. 366, no. 4-5, pp. 377-381, 2007.

[19] Q. D. Wang and M. Y. Tang, "New exact solutions for two nonlinear equations," Physics Letters A, vol. 372, no. 17, pp. 29953000, 2008.

[20] B. He, Q. Meng, and S. Li, "Explicit peakon and solitary wave solutions for the modified Fornberg-Whitham equation," Applied Mathematics and Computation, vol. 217, no. 5, pp. 19761982, 2010.

[21] J. Guckenheimer and P. Homes, Nonlinear Oscillations, Dynamical Systems and Bifurcations of Vector Fields, Springer, New York, NY, USA, 1999.

[22] J. S. M. El-Sayed and D. Kaya, "Exact and numerical traveling wave solutions of Whitham-Broer-Kaup equations," Applied Mathematics and Computation, vol. 167, no. 2, pp. 1339-1349, 2005.

[23] E. Yomba, “The extended Fan's sub-equation method and its application to KdV-MKdV, BKK and variant Boussinesq equations," Physics Letters A, vol. 336, no. 6, pp. 463-476, 2005.

[24] X. Z. Li and M. L. Wang, "A sub-ODE method for finding exact solutions of a generalized KDV-mKDV Equation with highorder nonlinear terms," Physics Letters A, vol. 361, no. 1-2, pp. 115-118, 2007.

[25] Y. Chen and B. Li, "General projective Riccati equation method and exact solutions for generalized KDV-type and KDVBUrgers-type equations with nonlinear terms of any order," Chaos, Solitons and Fractals, vol. 19, no. 4, pp. 977-984, 2004.

[26] Y. Chen, B. Li, and H.-Q. Zhang, "Symbolic computation and construction of soliton-like solutions to the (2+1)-dimensional breaking soliton equation," Communications in Theoretical Physics, vol. 40, no. 2, pp. 137-147, 2003.
[27] B. Li, Y. Chen, H. N. Xuan, and H. Q. Zhang, "On exact solutions of the nonlinear Schrödinger equations in optical fiber," Chaos, Solitons and Fractals, vol. 17, pp. 885-898, 2003.

[28] B. Li, Y. Chen, and H. Zhang, "Explicit exact solutions for new general two-dimensional KDV-type and two-dimensional KDV-BUrgers-type equations with nonlinear terms of any order," Journal of Physics A: Mathematical and General, vol. 35, no. 39, pp. 8253-8265, 2002.

[29] Z. Y. Yan, "Generalized method and its application in the higher-order nonlinear Schrodinger equation in nonlinear optical fibres," Chaos, Solitons and Fractals, vol. 16, no. 5, pp. 759-779, 2003.

[30] C.-L. Zheng and L.-Q. Chen, "Some novel evolutional behaviors of localized excitations in the boiti-leon-martina system," International Journal of Modern Physics B: Condensed Matter Physics, vol. 22, no. 6, pp. 671-680, 2008.

[31] A. Y. Chen, W. T. Huang, and J. B. Li, "Qualitative behavior and exact travelling wave solutions of the Zhiber-Shabat equation," Journal of Computational and Applied Mathematics, vol. 230, no. 2, pp. 559-569, 2009. 


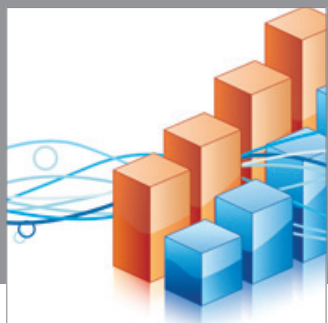

Advances in

Operations Research

mansans

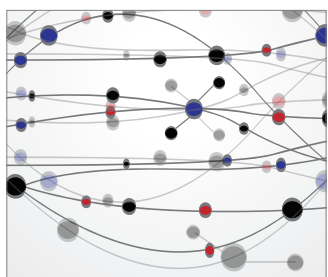

The Scientific World Journal
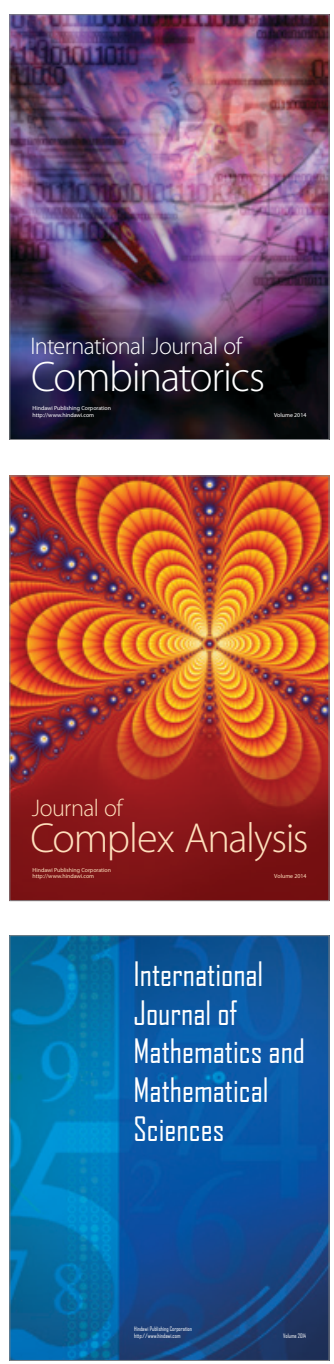
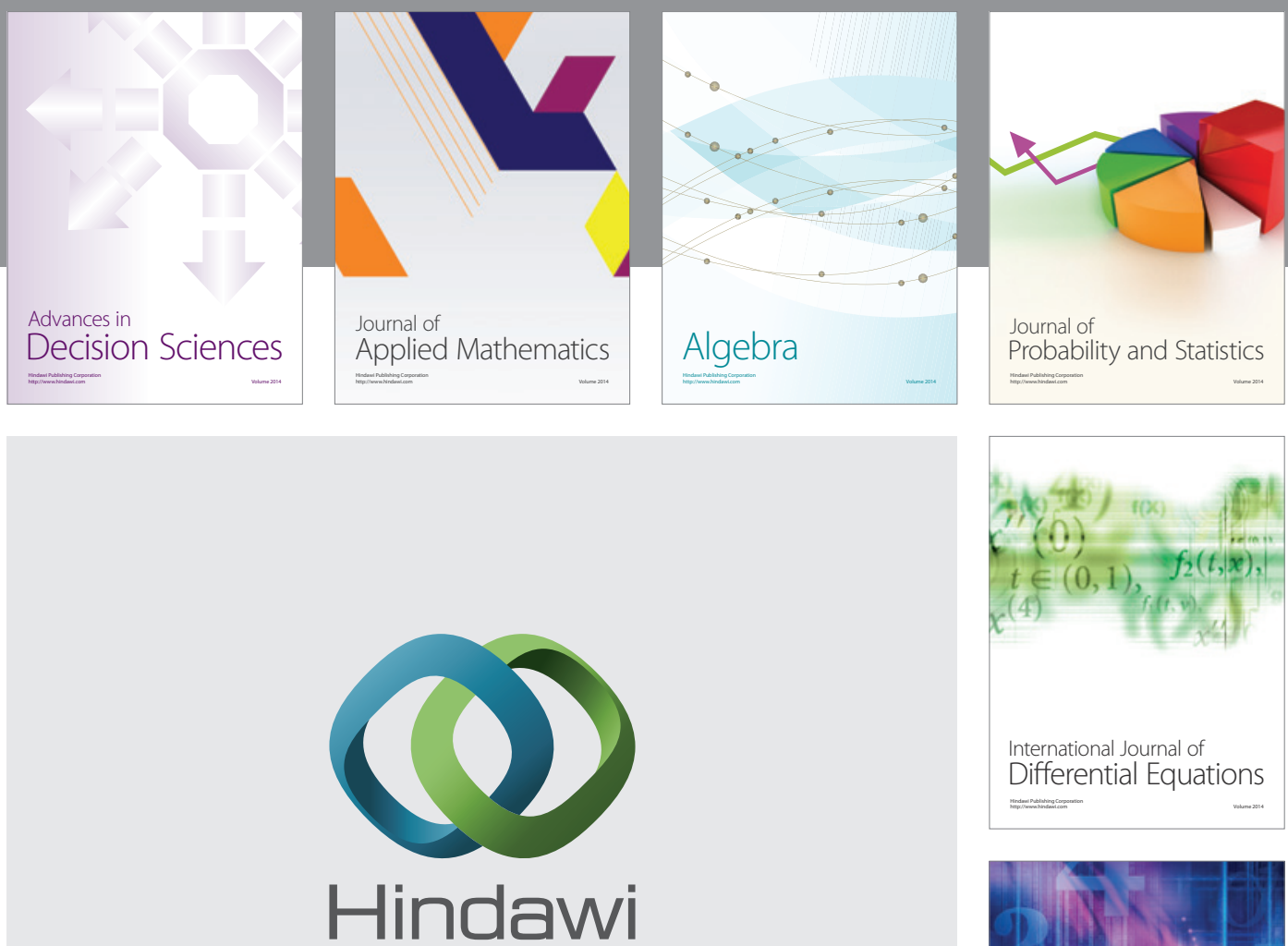

Submit your manuscripts at http://www.hindawi.com
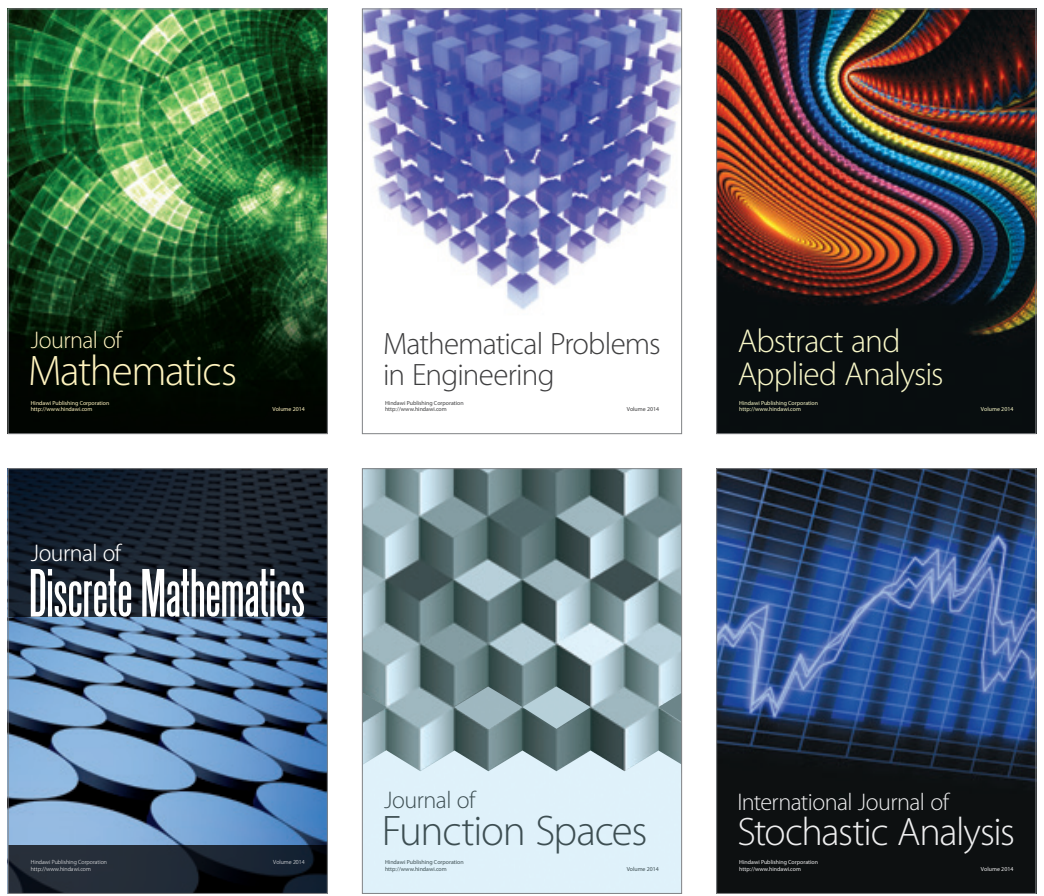

Journal of

Function Spaces

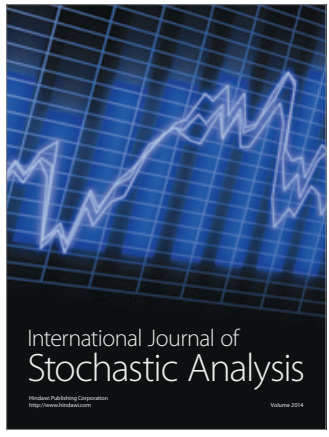

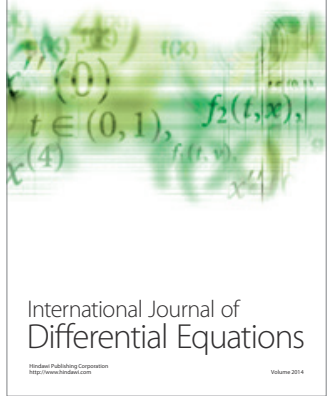
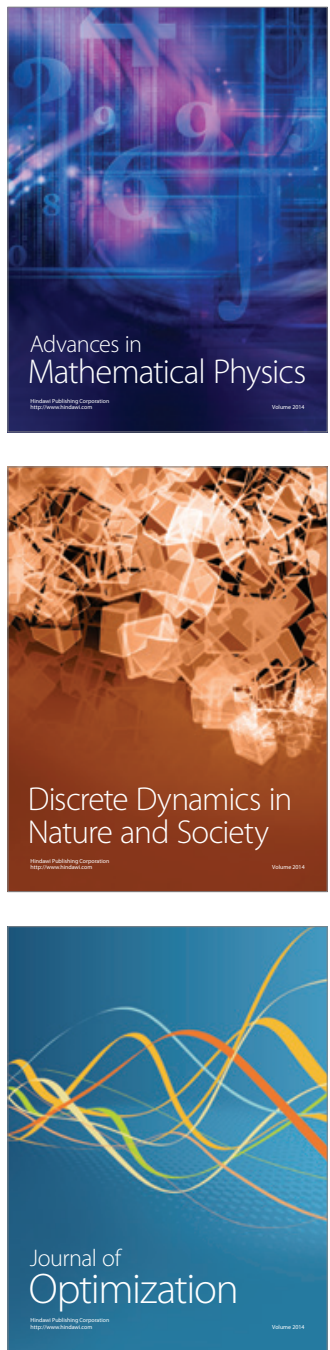